\title{
Research on License Plate Recognition Technology in ETC System
}

\author{
Wang Yi \\ Hubei Three Gorges Polytechnic, Yichang, Hubei, 443000
}

Keywords: license plate recognition technology; character segmentation; character recognition; character pattern matching

\begin{abstract}
License plate recognition technology process includes license plate location, character segmentation and character recognition. Firstly, according to the horizontal and vertical projection of the license plate, a rectangular region is found to locate the true position of the license plate, then the character binaries are transformed into recognizable black and white images, and the vehicle license plate numbers are segmented and normalized by character spacing. Finally, the characters are identified, and the extracted characters are inferior to the character template library which is set up in advance, and 0 means that the two are completely matched, saving each character that subtracts to 0 , which is the license plate you asking for.
\end{abstract}

\section{Design Principle}

The purpose of the license plate location recognition system is to obtain the license plate in the whole image region and to recognize the license plate accurately. It can recognize the license plate number from the static picture or the dynamic video, it is recognized by the character division of the different color area. The hardware part includes coil trigger device, capture device, central processor and so on, while the software part includes license plate region location algorithm, license plate character segmentation algorithm and optical character recognition algorithm. When the vehicle passes through the lane detection coil, the vehicle detection element is triggered, the system is informed of the vehicle passing, the image acquisition unit is activated to work, and the vehicle is captured as it passes the license plate recognition unit, beginning to analyze the image, locating the license plate in the whole image, dividing the single character of the license plate, and then recognizes the number of the license plate in turn and then recombines the output.

\section{Design Steps}

In the actual use of the license plate, the picture background of the car image is more complex and the light is not balanced. How to identify the license plate area in such an environment image is the key to the whole process of recognition. In the process of image acquisition, a large range of search is needed. Several candidate regions are selected and identified one by one. Finally, the license plate area is identified and segmented from the whole image area.

After locating the license plate area, the continuous license plate area is divided into a single character form, and these characters are recognized in turn. We usually separate character regions by vertical projection. This method is to use the projection between multiple characters in the vertical direction, take the minimum value of each character, and judge the range of the character, and see if the character size and character format of the license plate specification are satisfied.

In general, there are two main types of methods for character localization and recognition: One is to use neural network algorithm to identify, the other is to establish template library and use template matching method to identify. The algorithm based on artificial neural network can be divided into two methods: one is to extract the characters that to be processed, and then using these features to train the neural network. Another method is to directly use the Internet resources to help us recognizing the characters, input the selected eigenvalues through the network, and finally output the recognition results. The recognition rate of the license plate is related to the current status of the license plate and the clarity of the image taken by the camera. There are many current conditions of 
the license plate, such as the rusting of the license plate, the stain on the license plate, the partial exfoliation of the license plate, the blurring of the character font, the intentional occlusion of the license plate, the skew of the license plate, and so on. In the process of use, environmental brightness, camera equipment, speed and other factors will also affect the shooting. All these factors will affect the license plate recognition rate, which is the difficulty we have to overcome. In order to improve the recognition rate, Although new recognition algorithms are improving, it is also necessary to overcome the natural illumination conditions, which is conducive to the recognition of collected images.

\section{Detailed Design Steps}

Through the investigation and study of the current license plate in our country, it can be found that there are different forms of collocation of background color and character color in our country, generally there are five kinds of collocation: blue and white characters, yellow background black characters, white background red characters, black background white characters and white background black characters. To recognize characters, we need to separate them from the background image. For example, the most common blue-base white-letter license plate, under the blue channel, the license plate characters in the region can not be separated very well. There is not much difference between blue $(255,0,0)$ and white $(255,255,255)$ in blue channel, but not in $G, R$ channel or grayscale image. Therefore, the color image needs to be converted to gray image in recognition. The original color license plate image is shown in figure 1 and figure 2:

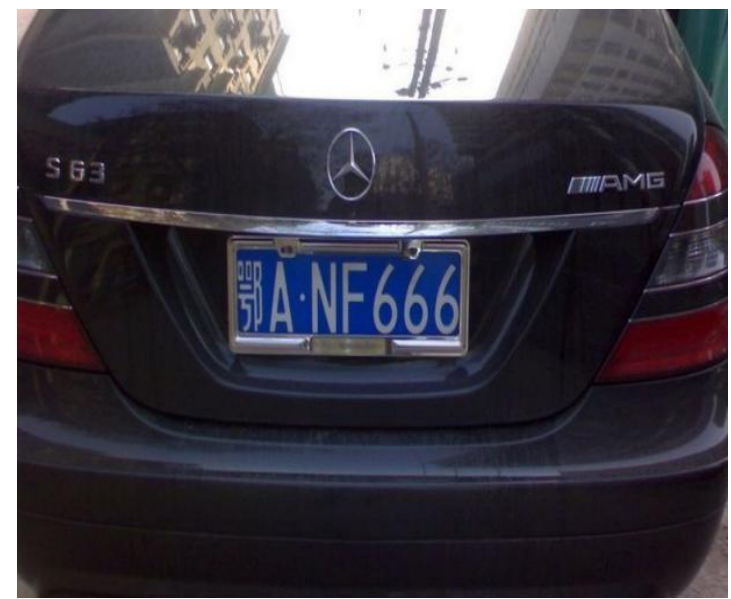

Figure 1 Original image

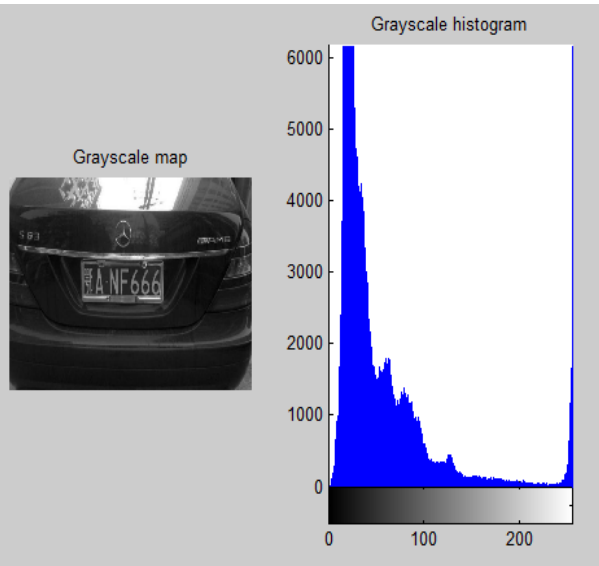

Figure 2 Grayscale image

The edges of an image have different brightness at both ends of the boundary. Using edge information can help us to extract the shape features of the target object. The main purpose of image edge detection is to enhance the image contrast. The main work is to make the license plate display more visible, so as to quickly select the license plate area, and then segment the license plate characters accurately. There are two main methods for image contrast enhancement: one is to correct the gray value when the gray value is not accurate; the other is to smooth the image when the edge of the image is rough.

The camera will be affected by the surrounding light when shooting the part of the license plate, which will cause the gray value of the image to be inconsistent with the actual situation, and the position deviation of the camera may also make the gray image of the vehicle incomplete. In order to reduce these unfavorable factors, we use the method of gray correction to make up for these errors. When the gray value is not enough, we can increase the grayscale level. In this way, the contrast of the image can be enhanced, and when the gray range is too small, the coverage of the gray value can be enlarged and the resolution of the image can be improved. Through the analysis of a lot of data, The gray value of the licence plate image varies roughly between the range of $\mathrm{R}=(50,200)$, and the image is usually shown as dark gray. In the absolute ideal state of image processing, the range of gray levels sought should be between the range of $S=(0,255)$. For this 
purpose, we can use the following formula to process it ${ }^{[1]}$ :

$S=T(r), r=[r \min , r \max ]$, Let $S \in[S \min , S \max ]$, where $\mathrm{T}$ is a linear transformation

$$
S=\frac{S \max -S \min }{r \max -r \min } \mathrm{R}+\frac{S \min \times r \max -r \max \times r \min }{r \max -r \min }
$$

If $\mathrm{R}=(50,200), \mathrm{S}=(0,255)$

$$
S=\frac{255}{150} \cdot R-\frac{255 \times 50}{150} \approx 1.7 R-85
$$

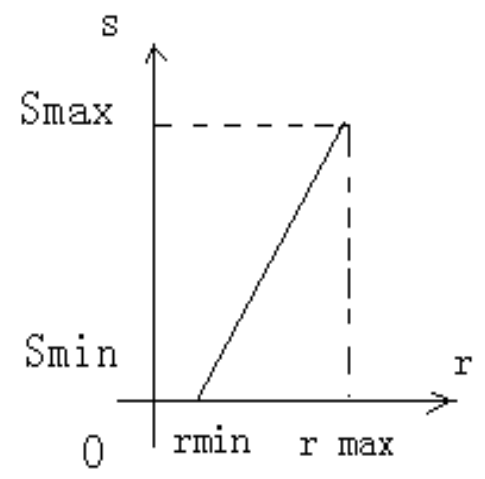

Figure 3 Gray scale linear transformation

Because of the presence of noise in the image, and the noise is mostly high frequency components. Therefore, we use low-pass filtering to filter noise interference, and we can reduce the noise interference by finding the mean value of the field near the reference point. We usually refer to this method as image smoothing. Choose a reference point with two neighborhood representations of 8 or 4 , as shown in the following two figures:

\begin{tabular}{|c|c|c|}
\hline 4 & 3 & 2 \\
\hline 5 & $\mathrm{i}, \mathrm{j}$ & 1 \\
\hline 6 & 7 & 8 \\
\hline
\end{tabular}

Figure 48 Domain Map

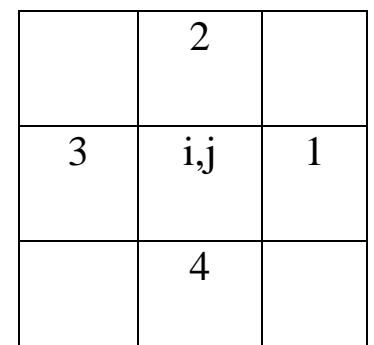

Figure 54 Domain Map

The respective neighborhood averages are

$$
g(i, j)=\frac{1}{M} \sum_{(i, j) \in s} f(i, j) g(i, j)
$$

The characteristic is that the value of $M$ near and outside the central pixel $f(i, j), M$ values can be 4 or 8 , depending on the selected neighborhood. Noise is often inextricably associated with the signal in the image. If the smoothing operation is not used properly, the contour of the image edge will be blurred. In order to overcome this disadvantage, we can set a threshold value, when the pixel 
value of a point is greater than the threshold value, the point can be expressed as the neighborhood average value, while when the pixel value is less than or equal to the threshold value, the original value will Keep the original value unchanged, and the effect of detail ambiguity will be reduced.

The license plate has a lot of features, the main features are: from the perspective of image processing, The license plate area is shown as a nearly horizontal rectangular frame, and the license plate numbers are arranged along the horizontal axis, and the spacing between them is the same. According to this characteristic, the image edge can be clearly reflected by the corresponding image transformation of the license plate. The Roberts operator can be used to extract the edges.

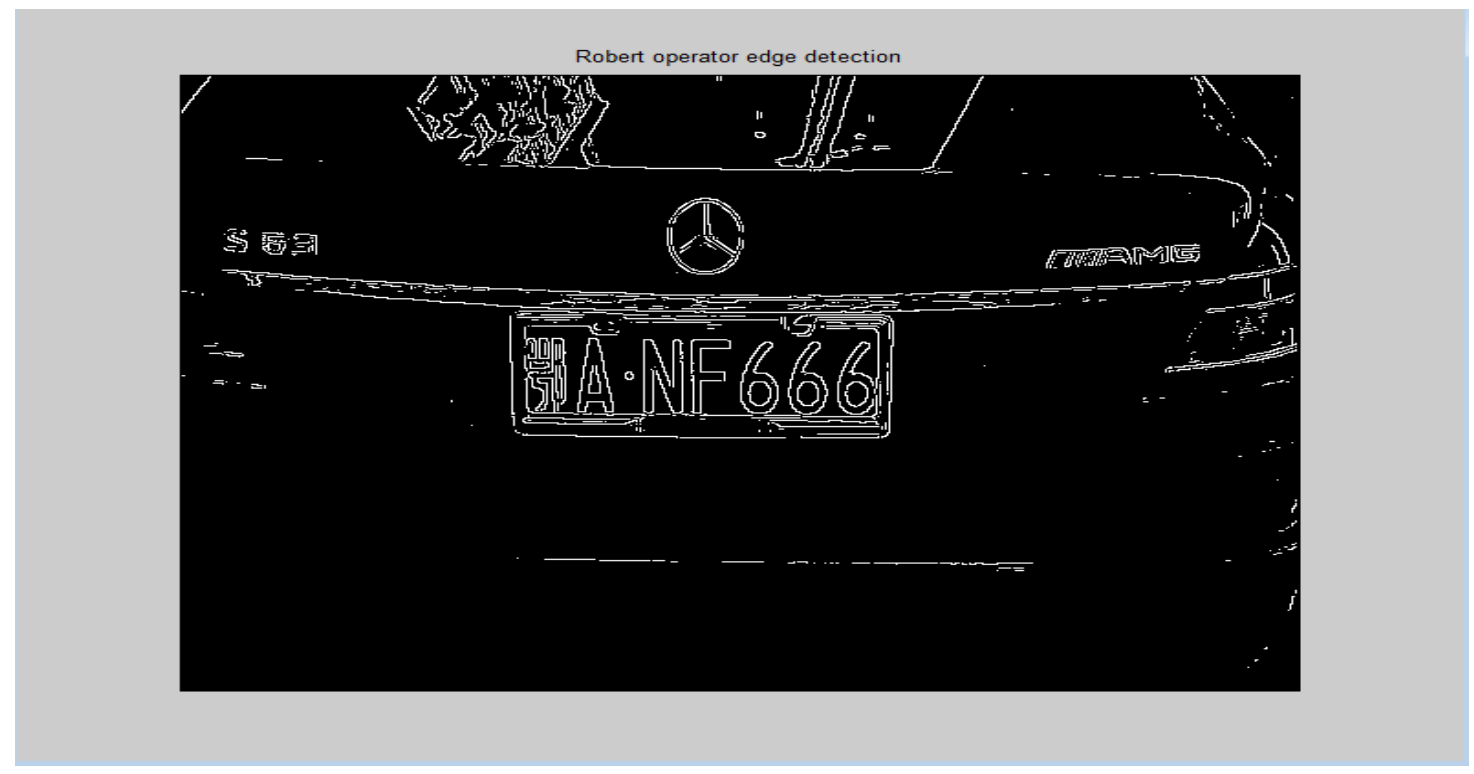

Figure 6 Roberts operator edge detection

The key of license plate recognition is to find the location position of the license plate and divide the character by one by one. First, the actual position of the license plate is searched in the gray map obtained first, the license plate image is separated from the background area, then the character of the license plate is read and recognized. Before recognition, the string is divided into single character first. In the original image, the original image is divided into a single character. The license plate image in the image is a very special subarea, the shape is similar to the rectangle, and because the license plate is firmly fixed, its position is relatively stable, which is also convenient for recognition, and because the color of the license plate and the color of the body are generally different, Therefore, it has different gray value with the surrounding environment variables, and there will be a mutation of the gray value between the edge and the outside. It can be conveniently located by the edge detection.

After the above processing, the license plate area has been roughly clear. At the same time, through the expansion operation, the edge of the license plate is thickened to make the edge line smooth. In this paper, mathematical morphology is used to solve the problem. Its main function is to analyze or process the shape and structure of the image. Mathematical morphology can preserve the morphological characteristic value of the image and remove the interfering object. In this example, the expansion and closure operations are combined, and the final goal is to remove the irrelevant objects in the image.

When we know the color of the license plate in advance, for example, the blue plate, we find out the RGB chroma range corresponding to the blue, and find the blue area of the image point by the column scan method. Finally, after locating and shearing, we get the license plate area that are needed. After cutting the license plate image, there still exists noise interference in the image, and the target object is accurately extracted from the image. The simplest way is to set the threshold. We assume that the threshold is expressed as $\mathrm{T}$, which can divide the image data into two parts. The method is to find the target pixel point and the adjacent pixel point area to create a template, and replace the original pixel value with the average value of the template area. 


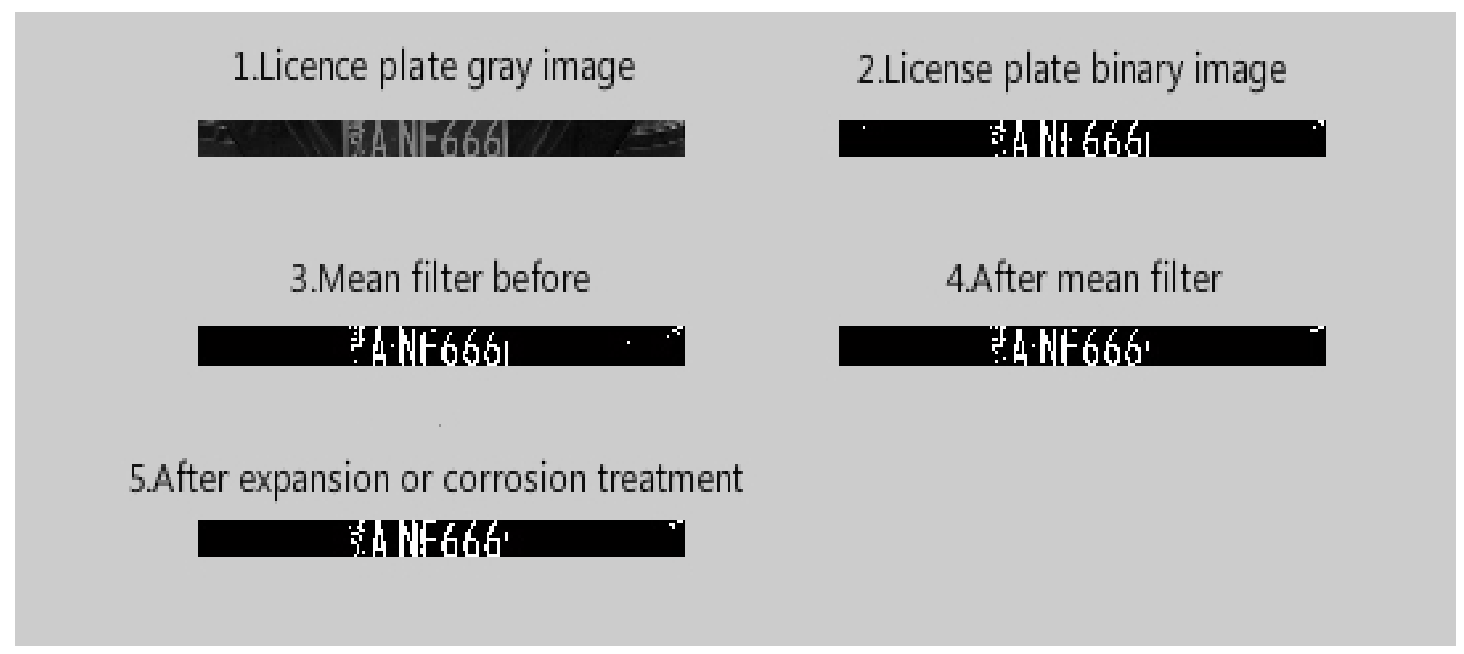

Figure 7 License plate processing

Character segmentation plays an important role in the whole recognition process. The method is to locate the position of the license plate, then segment the license plate. Finally, the result of segmentation is recognized. Real license plate character spacing is still large, and there is no overlap between the two characters. We directly check the string in the two value image of the license plate before cutting, and if the length of the character is less than a threshold, it is a single character; when the length of the character is greater than this threshold, it is two connected characters. The two characters need to be separated.
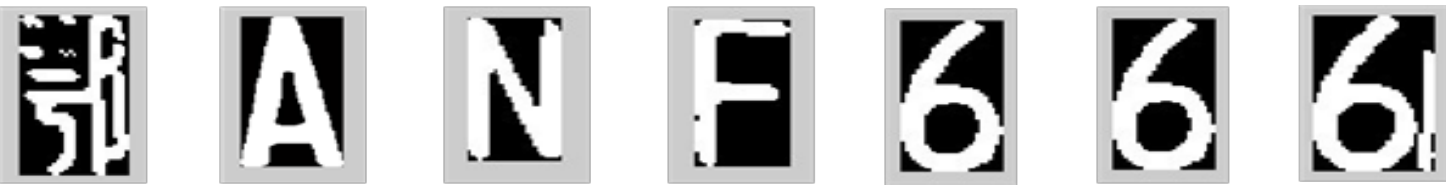

Figure 8 Seven characters split out

The characters are processed one by one, and the license plate is restored at last. This requires the normalization operation, which is completed after processing and subsequent steps.
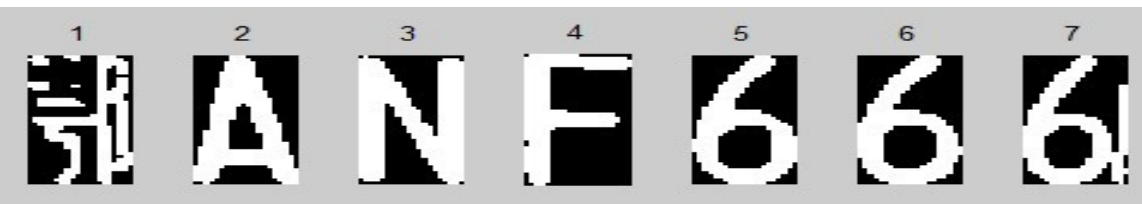

Figure 9 Normalized display of seven characters

There are two methods for the character recognition of the license plate: the template matching algorithm and the artificial neural network algorithm. The work flow of the template matching algorithm is: first, the character of the license plate is two values, then the characters are matched with the information stored in the template library, and the results are output, because the characters in the template library are usually $\mathrm{A} \sim \mathrm{Z}$, and the numbers are $0 \sim 9$, the Chinese character is only the province, the municipality directly under the central government, the army, the police, the template library is not very large, so this method is relatively simple to use. And when the character image has the defect or the stain interference, it can also maintain a high recognition rate. It is the template match. The advantage of matching method is so obvious that it has become the mainstream method of license plate recognition.

The template matching algorithm is the most representative method of image recognition. This method is to compare the feature values extracted from the $f(I, J)$ to the corresponding template library T (I, J), and calculate the similarity between them. Among them, a large degree of similarity between two images means that the relationship between them is more closely related to the same class. However, in general, the matching conditions for each image are different, and the noise 
interference is larger.If there is a reading error in image preprocessing or normalization processing, it will also result in the image position deviation or pixel gray value deviating from the true value. When we design the template, we should be able to recognize each feature of the image region and the difference between each other, and select the invariant quantity to analyze when selecting the feature value, so we can improve the matching degree of the template ${ }^{[5]}$.

We use subtracting operations to find the similarity between the license plate characters and the characters stored in the template library and identify the most similar result output. License plate characters are only seven bits; the character arrangement has its fixed rule. The first character is usually a Chinese character, and the Chinese character has its own rules, generally Abbreviations for provinces, municipalities directly under the Central Government, plus military or police cards. Chinese characters are followed by letters and letters are followed by numbers. We can set up the character template library based on the rule of character arrangement. The license plate is also used in less than 50 Chinese characters, 26 basic capital English letters, 10 decimal basic numbers, and the template is more convenient. The method is to match the license plate characters with the template library on the basis of building the template library in advance. Subtract the obtained license plate characters and the characters in the template library, and if 0 is obtained, the match between the two characters is completed. Saving each character that subtracts to 0, which is the plate number you are looking for ${ }^{[4]}$.

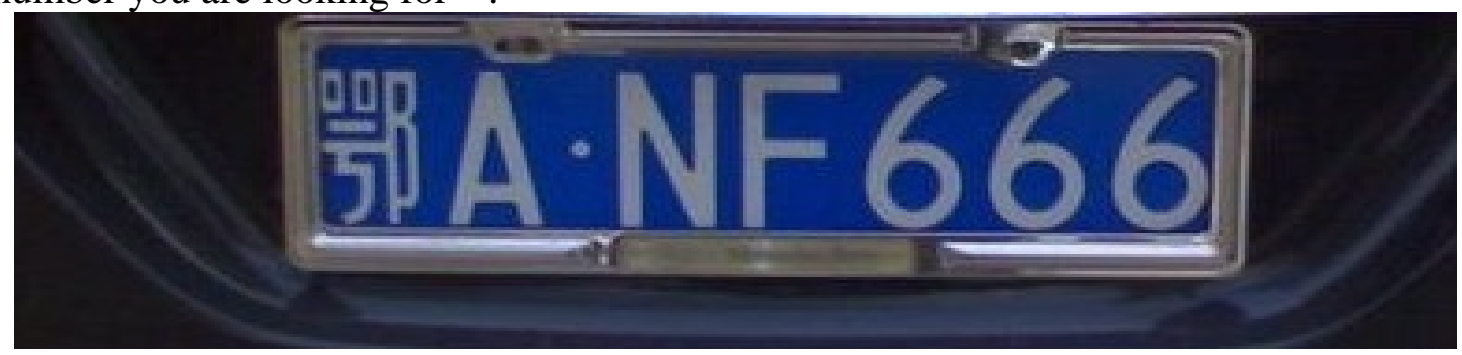

Figure 10 Recognition of the license plate results

\section{Acknowledgement}

Fund Project: Yichang City Applied Basic Research Project, "Research on vehicle license Plate pattern recognition in Expressway ETC system", project number: A14-302-b02

\section{References}

[1] Canny. A Computational Approach to Edge Detection[J]. IEEE Tram, Pattern Analysis and Machine Intelligence.2008

[2] M. Fathy, M.Y.Siyal. Image Segmentation using Probabilistic Fuzzy C--Means Clustering [J]. 2007

[3] Otsu N. A threshold selection method from gray level histograms. IEEE Trans SMC.2010.9

[4] Igor Kuzmanovski, Marjana Novic, Counter-propagation neural networks in Matlab.2007.4

[5] David A. Hens her. Electronic Toll Collect ion.Trans.Res.1990(2) 